\title{
Q3M - QoS Architecture for Multi-user Mobile Multimedia Sessions in 4G systems
}

\author{
Eduardo Cerqueira ${ }^{1}$, Luis Veloso ${ }^{1}$, Augusto Neto ${ }^{1}$, Marília Curado ${ }^{1}$, Paulo Mendes ${ }^{2}$, \\ and Edmundo Monteiro ${ }^{1}$ \\ ${ }^{1}$ University of Coimbra, Pinhal de Marrocos, 3030-290 Coimbra, Portugal \\ \{ecoelho, lmveloso, augusto, marilia, edmundo\} edei.uc.pt \\ ${ }^{2}$ DoCoMo Euro-Labs, Landersbergerstr, 312, 80687 Munich, Germany \\ mendes@docomolab-euro.com
}

\begin{abstract}
Fourth generation systems (4G) will provide multimedia group communication sessions to multiple mobile users with distinct requirements. This way, it is expected the control of the quality level, connectivity and ubiquitous access for multi-user multimedia sessions across heterogeneous and mobile networks with seamless capability. This paper analyses the requirements of a control architecture to provide Quality of Service (QoS), connectivity and seamless handover management for multi-user sessions in 4G systems, and introduces the QoS Architecture for Multi-user Mobile Multimedia (Q3M) proposal. In addition, simulation results present the efficiency of this approach concerning the session setup time and packet losses during handover.
\end{abstract}

Keywords: Multi-user Multimedia Sessions, Seamless Mobility, Quality of Service, Heterogeneous Mobile Networks.

\section{Introduction}

Fourth generation systems are expected to allow mobile users to access group communication sessions over heterogeneous wired and wireless networks with QoS support. Examples of these sessions are IPTV, video streaming, push media and other multicasting multimedia-alike sessions. Since the content is simultaneously destined to multiple users, this type of sessions are called multi-user sessions. Based on wellknow codecs, such as MPEG4, multi-user sessions can also be scalable. Each scalable session can be composed by a set of flows, with well-defined priorities, rates and QoS requirements. Hence, the importance of each flow must be used to adapt the overall quality of the session to the capability of different networks service classes.

The delivery of multi-user session may be done through different wired and wireless access technologies [1]. This heterogeneity may go from cellular and wide area access technologies (e.g., Worldwide Interoperability for Microwave Access (WiMAX)), to local area access technologies (e.g., Wireless Local Area Networks (WLAN)), and broadcast media such as Digital Video Broadcasting-Terrestrial (DVB-T) and DVB-Handheld (DVB-H). This diversity requires an infrastructure to 
reduce operational costs and enhance efficiency in the usage of network resources, where these requirements are satisfied by an all-IP 4G mobile system.

In addition to the access technologies heterogeneity, the $4 \mathrm{G}$ systems are expected to be heterogeneous also at the network layer, due to the diversity of connectivity control mechanisms and different address realms. For instance, in terms of unicast mobility management, the use of Hierarchical Mobile IP (HMIP) allows users to use a sequence of two global IP addresses, inside and outside each access-network. In the field of IP multicast, which is the most suitable technology to distribute multi-user sessions, there are already several address realms associated with multicast models such as, the Any-Source Multicast (ASM), Source-Specific Multicast (SSM) and Small-Group Multicast (SGM).

However, to allow the distribution of multi-user sessions throughout heterogeneous access and transport technologies, it seems to be mandatory to cluster homogeneous network devices into domains (named clusters in this paper) and to create of open interfaces between such clusters and existing standards. Therefore, a control architecture should be built as modular and decentralized as possible. The modularization facilitates the inclusion of emerging technologies, while the decentralization permits a higher scalability. Both of these characteristics might be sustained by an edge-networking approach in which the functionality of each cluster is controlled by a group of organized edge devices.

Since each cluster may implement a different connectivity scheme (e.g., IP unicast, IP multicast, Multimedia Broadcast Multicast Service (MBMS) or any type of Layer 2 connectivity), the architecture must control the multi-user session connectivity cluster by cluster. This functionality allows the session continuity independent from the connectivity scheme offered inside or between clusters. Hence, each multi-user session must have a global identifier, independent from hosts location or IP address. Furthermore, in order to support emerging mobile-aware sessions, to increase the satisfaction of the users and to avoid the interruption of sessions, an architecture must provide ubiquitous access, QoS and mobility control across heterogeneous clusters with no perceived service degradation for the user.

From the QoS point of view, different QoS models (e.g., such as Differentiated Service (DiffServ), IEEE 802.11e and IEEE 802.16) can be implemented by clusters to provide QoS assurances for multi-user sessions along end-to-end heterogeneous paths. Hence, inside or between clusters, QoS mapping procedures must be done to map the requirements of the session into the appropriated service class. Since QoS mapping alone is not sufficient to assure the quality level of sessions, due to the use of different QoS models, class of services with distinctive configurations and available bandwidth capacities, QoS adaptation schemes must be used to avoid the session blocking and to keep those sessions with acceptable quality level, independently of the movement of users or even re-routing events (caused by a link or network agent failure). From the mobility point of view, an architecture must support inter and intra-cluster handovers with seamless capability. For instance, by using caching and buffering mechanisms to reduce packet losses during handover and by reserving network resources in advance to allow a faster session re-establishment.

Summing up, an IP-based control architecture for multi-user multimedia sessions must allow senders to offer their content and receivers to access them ubiquitously. It must also provide open interfaces, QoS, connectivity and seamless mobility control. 
This paper first analyzed the requirements for a control architecture for multi-user sessions over an IP-based 4G mobile system. Following, the QoS Architecture for Multi-user Mobile Multimedia (Q3M) proposal is presented, together with an illustration of its functionalities and an insight to its performance.

The next section presents relevant related work. The Q3M architecture is described in Section 3. Section 4 illustrates an example of the Q3M overall functionality. An evaluation is shown in Section 5. Conclusions are summarized in Section 6.

\section{Related Work}

There are IETF standards that address some of above mentioned needs, such as the DiffServ model to provide QoS assurance, the Next Step in Signaling (NSIS) QoS Signaling Layer Protocol (NSLP) for signaling resource reservations for unicast sessions, the Session Initiation Protocol (SIP) to control the access to announced sessions, the Protocol Independent Multicast for the SSM (PIM-SSM) for packet distribution to groups of users. The MIP, HMIP and the Fast Handovers for MIPv6 (FMIPv6) to control handovers in unicast networks. FMIPv6 adds seamless experience to the users, by transferring and buffering packets from old to new accessrouters. The Context Transfer Protocol (CXTP) can reduce latency and packet losses, by transferring the session-context among access-routers. However, each of these standards does not cover by itself all aspects for the development of an architecture to provide connectivity, QoS, and seamless handover for multi-user sessions.

In addition to the IETF solutions, architectures for QoS, mobility and heterogeneous systems have been proposed. The FAMOUS architecture [2] aims to support broadband services to fast moving users. This proposal only supports singleuser sessions and uses a tunnel-based approach to control connectivity, restricting network operations. The MUSE project [3] proposes QoS architecture for stationary users to access broadband services in unicast environments. However, mobility is not supported and the QoS control is based on a static guideline mapping approach to map the session requirements into four proprietary classes, which reduces the system flexibility. The DAIDALOS project [4] provides an architecture that supports the distribution of unicast and multicast sessions across heterogeneous mobile systems. However, the DAIDALOS architecture uses a centralized approach to control perflow QoS resources and multicast tree creation, reducing thus the system scalability. The EuQoS project [5] supports a QoS architecture based on brokers to control network resources and an Application Layer Multicast (ALM) to distribute packets using unicast connections. However, it does not take heterogeneous connectivity scheme or IP multicast into account. Instead, it uses an ALM that requires extra modules on the end-hosts and does not avoid sending redundant data over the same physical link. A 4G architecture to provide QoS, resource reservation and mobility is introduced in [6]. This architecture uses QoS brokers to control network resources for the sessions and hierarchical FMIPv6 schemes to provide the continuity of unicast sessions, but it does not avoid packet losses nor assure seamless experience for ongoing sessions. This architecture does not also take the use of heterogeneous QoS models into account and the packet differentiation in wireless links is not supported. 
From the related work analysis it is evident that the IETF solutions are not sufficient to provide QoS, connectivity and seamless mobility for multi-user session. Additionally, several architectures do not support multicast sessions and only control network resources and handover in unicast networks. Moreover, the use of centralized approaches to control QoS and mobility reduces the flexibility and scalability of the system. To overcome the identified limitation, the University of Coimbra is working together with NTT DoCoMo Euro-labs in an all-IP 4G control architecture called QoS Architecture for Multi-user Mobile Multimedia (Q3M).

\section{The Q3M Architecture}

The Q3M architecture controls multi-user sessions across heterogeneous clusters through a modular integration of three components: the Multi-User Session Control (MUSC) [7], the Multi-service Resource Allocation (MIRA) [8] and the Cache-based Seamless Mobility (CASM) [9]. The coordination of these components provides ubiquitous access, resource reservation, QoS mapping, QoS adaptation, connectivity and seamless mobility control for multi-user session in $4 \mathrm{G}$ systems.

MUSC, MIRA and CASM use interfaces to allow a tighter communication between themselves and external solutions, such as SIP, Session Description Protocol (SDP), PIM-SSM, Internet Group Management Protocol (IGMPv3), Multicast Listener Discovery (MLDv2), DiffServ elements and Wireless Controllers. For instance, the interaction between MIRA and Wireless Controllers, such as used by WiMAX or IEEE 802.11e [10], allows QoS support in wireless links. The interface among CASM and Wireless Controllers gives seamless support for mobility control functions accomplished by the former. These interfaces are represented in Fig. 1.

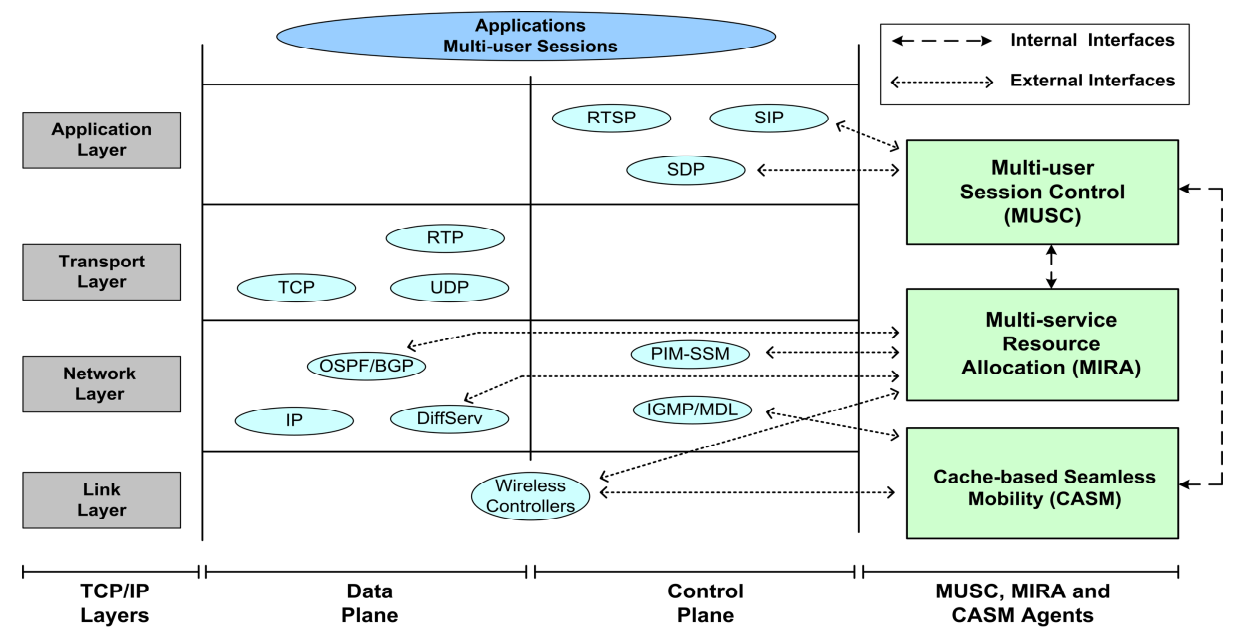

Fig. 1. Q3M components in a TCP/IP stack view

The referred components are implemented in an element named Q3M-Agent (Q3MA). A Q3MA is called access-Q3MA when located at wired or wireless 
access-routers. Furthermore, Q3MAs can have distinct roles in different edges for different sessions: in an edge-router, a Q3MA is called an ingress-Q3MA for sessions whose traffic is entering the cluster in that edge element, or egress-Q3MA if the traffic is leaving the cluster.

\subsection{Q3M Components}

This sub-section details the Q3M components.

\subsubsection{Multi-user Session Control}

MUSC manages the mobility of multi-user session, as well as the ubiquitous access of users to those sessions. The cooperation between MUSC agents allows QoS mapping, QoS adaptation and connectivity of multi-user sessions along heterogeneous end-toend path. The ubiquitous access control is done in the access-agent through an interface with SIP and CASM to allow fixed and mobile users to access multi-user sessions respectively. The interface with CASM provides the control of mobility with a seamless capability, by allowing sessions to be configured in advance on new paths and by reducing the number of packet losses during handover. In ingress and egressagents, the QoS mapping is based on the association between the session quality requirements and the available network services provided by MIRA. If such mapping is not optimal, for instance due to a congestion period, the session is not blocked but a QoS adaptation to the current network conditions is done. The adaptation mechanism can request the allocation of extra resources to the selected service class, the mapping of flows into another class, or the dropping/joining of low priority flows of sessions.

If the ingress or egress-agent is in the frontier between unicast and/or multicast address realms, MUSC configures its connectivity translator mechanisms, allowing multi-user sessions to be distributed independently of underlying connectivity technology. In unicast-aware networks, the packet distribution is handled in a multicasting way to save network resources. The packet duplication is performed near to the receivers (application layer multicasting). The coordination of a chain of connectivity translators supports any layout of heterogeneous networks and end-hosts.

A receiver-driven and source-initiated protocol, called MUSC-P, is used to exchange information between MUSC agent using a soft-state approach to maintain per-session and per-flow state. MUSC-P is being specified based on the NSIS framework, in which it can be included as an extra NSLP. MUSC-P is a receiverdriven protocol because it is triggered at access-agents. It is source-initiated since MUSC starts the configuration of its agents at the agent nearest to the source, or at the first agent, in the path towards the source, discovered which contains the requested session. This functionality gives support for building QoS-aware distribution trees as well as it reduces the session setup time and signaling overhead to establish sessions.

\subsubsection{Multi-service Resource Allocation}

MIRA aims to build QoS-aware distribution trees associated with multi-user sessions in environments with asymmetric routing. MIRA controls network resources for unicast and multicast sessions in a per-class basis, where it is done inside and between networks based on DiffServ model and Service Level Specifications (SLS) respectively. MIRA also provides QoS guarantees on the wireless link through the 
interaction with a wireless resource allocation controller. Therefore, MIRA gives support for QoS mapping and QoS adaptation operations and assures the QoS level for each flow of a multi-user session controlled by MUSC.

In multicast-aware networks, MIRA follows the SSM model for controlling multiuser distribution and it also allocates a SSM channel (e.g., source and multicast group) to be used for each flow of a session. In unicast-aware networks, a pair of IP unicast addresses and transport ports is allocated to identify each flow of a session. Based on the information about the distribution channel identifiers provided by MIRA, MUSC controls the session connectivity independently of the address realm implemented along the end-to-end session path. In SSM-aware environments, MIRA provides support so that the multicast routing protocol (e.g., PIM-SSM) creates QoS-aware multicast trees taking into account the path from the sender to the receiver. This is done through updating the Multicast Routing Information Base (MRIB) with the QoS path during the resource allocation work, enabling thus PIM-SSM to create the trees without suffering with asymmetric routes.

The control information is supplied to MIRA agents through the MIRA Protocol (MIRA-P), which is being specified as a new NSIS QoS-NSLP with multicast support. MIRA-P operates edge-to-edge and controls the state maintenance by softstate: periodically signaling the paths with the amount of state to be kept and to collect network resource capability information for admission control. MIRA does not compromise inter-network links performance since it configures the network resources based on SLS, without requiring signaling exchanges on those links.

\subsubsection{Cache-Based Seamless Mobility}

CASM supplies users with a seamless moving experience among access-Q3MAs belonging to the same neighbor-clusters. This is done based on the use of caches in access-Q3MAs and buffers in mobile devices to reduce packet loss. In the presence of a handover, the data in the buffer of the mobile receiver will continue to be read by the application. When the handover is resumed, the mobile receiver updates its buffer by fetching the missing packets from the cache in its new access-Q3MA. The latency experimented by the user depends on the size of the buffer and handover duration.

In its enhanced configuration, CASM uses of an interface to receive information concerning the new probable access-Q3MAs to which the user can move, being the number of access-agents dependent on the prediction granularity [11]. Upon receiving information about the candidate access-Q3MAs, the session context is transferred among access-Q3MAs. The use of an interface with mobility prediction controllers together with CASM context transfer mechanism allows the session setup in advance by interacting with MUSC, increasing the assurance of low latency.

CASM-P is the CTXP-based signaling protocol used to transfer the context of multi-user sessions between old and the predicted access-Q3MAs. It also collects information about the capability provided by the latter. At the old access-Q3MA, the probed information, combined with knowledge regarding the access technologies, such as signal-to-noise ratio, gives support to the handover decision. After handover, the interaction between CASM and MUSC allows the release of the session resources on the old path and on the predicted paths that the mobile device is not going to use (if no other users are subscribed to the same flow of the session). 


\subsection{Q3M Interfaces}

Interfaces are used to exchange control information between Q3M components and existing solutions as follows.

The MUSC-CASM interface is used in access-Q3MAs to support seamless mobility. When a new session is accepted by MUSC, CASM is triggered to create a cache for the new session (if the session does not exist). During handover, CASM triggers MUSC in predicted access-agent(s) to setup the session and to collect information concerning the capability and connectivity of the new paths. At the previous access-agent, the interaction between CASM and MUSC allows the release of resources associated with the session on the old path.

The MUSC-MIRA interface is implemented in all Q3MAs providing end-to-end connectivity and QoS support over heterogeneous clusters. MUSC triggers MIRA by querying it about the QoS characteristic of the cluster network services. After selecting a network service for the flows of the session, MUSC requests to MIRA the allocation of the resources required by each flow. As a response, MUSC gets from MIRA information about the unicast flow or multicast tree to which the flow was associated inside or between clusters. When there are no more users in a flow of a session, MUSC triggers MIRA to release the resources associated with each flow.

MUSC interacts with SIP to allow users to join or leave a multi-user session. Applications compose a SIP/SDP message to join announced sessions. This message is received by a SIP-proxy in the access-cluster, which can accomplish some authentication, authorization and accounting procedures. After performing its operations, the SIP-proxy forwards the session information to the receiver's accessQ3MA based on SIP Location Servers. The reception of this information activates the MUSC access-control. After the conclusion of the MUSC operations, the application is informed, by means of a SIP/SDP message, that its request was accepted or not. For multicast-aware devices placed in multicast-aware access-clusters, MUSC also informs the receiver about the multicast channel to be joined by each session-flow (as a consequence, IGMPv3 or MLDv2 messages are sent to the access-router).

MIRA interacts with DiffServ elements to collect information about the network services (loss, delay, jitter, and available bandwidth) by checking the Per-HopBehaviour (PHB) table on each network node from the ingress to egress. Based on the session-flow information provided by MUSC (flow rate and network service to be used), MIRA adjusts the bandwidth share of the PHB of the selected network service in each router of the cluster ingress-egress path. The interface between MIRA and wireless technologies is being considered, such as 802.11e and 802.16. Therefore, MIRA is configured with information about the wireless service class capabilities, allowing QoS support on the wireless interface.

The MIRA-PIM-SSM interface allows the former to update the MRIB on each router, or to directly configure the SSM channel on outgoing interfaces.

The interface CASM-IGMPv3/MLDv2 allows users in multicast-aware clusters to join the multicast channels allocated for each flow of a session in new access-agents. 


\section{Example of Q3M Overall Functionality}

Fig. 2 shows the interaction of the Q3M components to setup a session $(S 1)$ in fixed and mobile scenarios. Therefore, the application on $R 1$ uses a SIP/SDP message to subscribe the announced session (step $i$ ). The SIP-proxy forwards the message to the receiver's access-Q3MA based on previous registration. Afterwards, the MUSC access-control is notified and triggers MUSC-P in Q3MA-12 to send a message towards the source with IP Router Alert Option enabled. This message is received by the Q3MA closest to the source of the signaled path, or by the first Q3MA, in the path towards the source, found with the requested session, as happens in Q3MA-33.

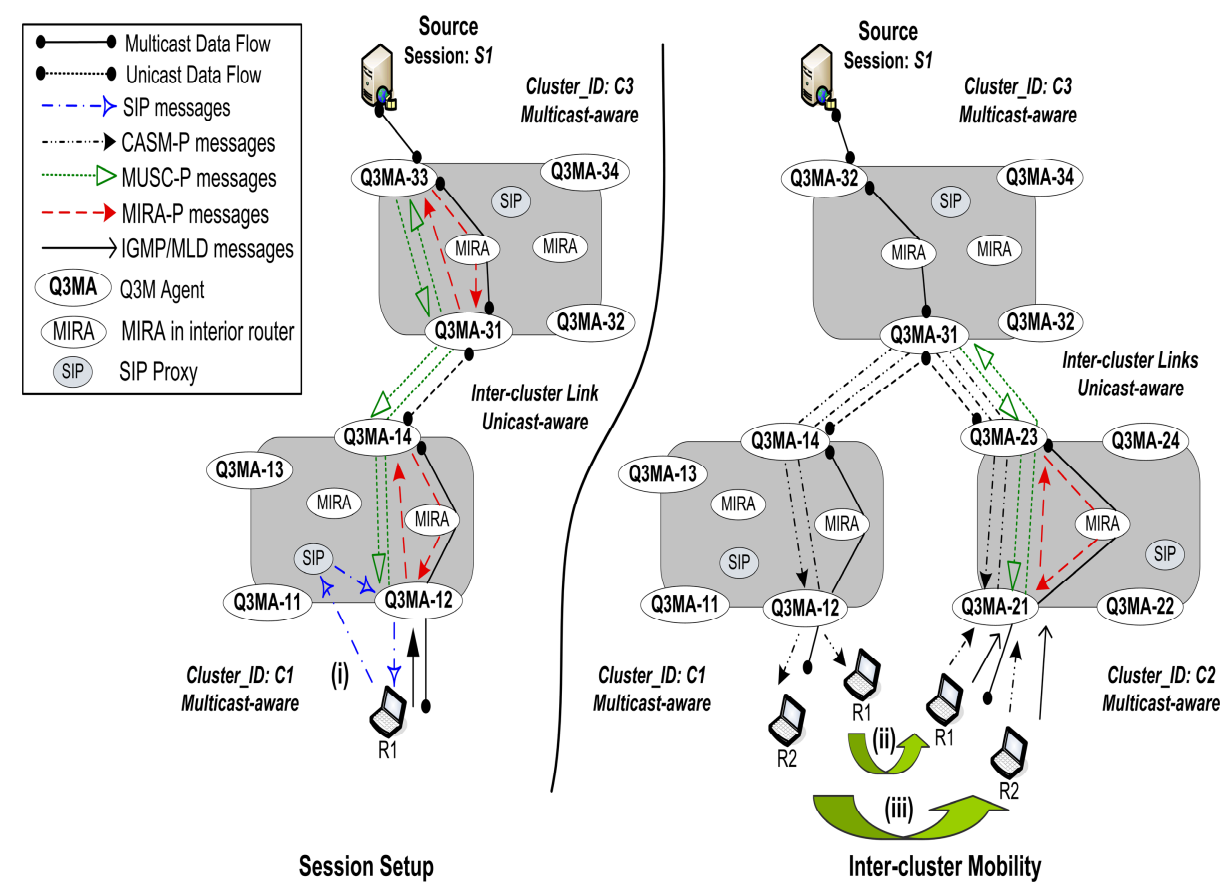

Fig. 2. Example of Q3M Functionality

In Q3MA-33, MUSC triggers MIRA to query about network services in the cluster path towards Q3MA-12. Based on the response, MUSC selects the preferred service class and requests MIRA to configure the required bandwidth. After admission control, MIRA updates the resources of the requested QoS class and configures the multicast (configuring the MRIB) on the path towards the egress Q3MA-31. When all intra-cluster operations succeed, MIRA informs MUSC about the multicast SSM channel allocated for each flow of the session. This information allows MUSC to start translating all incoming packets of $S 1$ to the indicated intra-cluster multicast trees.

After the operations in Q3MA-33, MUSC in Q3MA-31 is triggered by MUSC-P, updating its translation state about the SSM channel used for the flows of $S 1$ in $C 3$. 
The interaction with MIRA occurs as described before. Since Q3MA-31 is an egressagent, PIM-SSM is triggered to create multicast trees for each flow of the session in the QoS-aware reserved-path. MIRA also collects QoS information by checking the inter-cluster SLSs, instead of querying the network. Since unicast is used between clusters, MIRA gets from the SLS the unicast IP address of the next Q3MA (Q3MA14) as well as a pool of available ports. Based on this connectivity information, a pair of source and destination ports is allocated for each flow of $S 1$. It allows MUSC to translate all packets coming from the intra-cluster to the pair of source and destination IP addresses and ports allocated for each flow between $C 3$ and $C 1$.

When the MUSC-P response message reaches the access-Q3MA-12, MIRA is required to control the multicast tree creation inside $C 1$ and to inform the available service class on the wireless interface. After MUSC QoS and connectivity control operations, MIRA is triggered to configure the required bandwidth in the selected wireless service class. Afterwards, MUSC interacts with CASM to activate/update the cache for the session and gives information concerning session-context. Finally, MUSC sends a correspondent successful SIP message to the receiver. In a multicast access-cluster, MUSC includes, in the SIP response message, information about the SSM channel to be joined by using IGMPv3 or MLDv2.

The situation on the right side of Fig. 2 represents the inter-cluster mobility, in which R1 (step ii) moves away from the access-Q3MA-12. Upon detection the next probable access-Q3MA to which the receiver is moving, CASM is triggered by the mobility prediction controller and receives information about the IP address of the new access-Q3MA (in this case Q3MA-21). After the handover prediction, CASM signals the predicted Q3MA with session-context information. In Q3MA-21, CASM triggers MUSC, which configures the session on the new path by interacting with MIRA for the reservation of network resources. The MUSC-P messages and procedures are performed only between Q3MA-21 and Q3MA-31 and not end-to-end, since the session is already active in the Q3MA-31. In Q3MA-21, CASM configures a session cache to avoid packet losses. After this, a reply is sent to CASM in Q3MA-12.

Based on the QoS configuration of the session on the new path and the signal-tonoise ratio, CASM in Q3MA-12 takes a handover decision to Q3MA-21. Hence, CASM informs MUSC about the handover, allowing MUSC to adjust the number of receivers and to possibly remove the state of the session. In this case, no state is removed, since $R 2$ is still subscribed. During handover, the application consumes packets from the $R l$ buffer while packets continue to arrive to the cache in Q3MA-21. After attaching to the new access-Q3MA, CASM sends a fetch message to request the missing packets and to sync the packet reception with the cache in Q3MA-21. Furthermore, CASM triggers the IGMPv3/MLDv2 to join the new multicast channel allocated to the flow of the session.

After the mobility of $R 1, R 2$ (step iii) also moves away from the Q3MA-12. In Q3MA-12, the mobility prediction controller notifies CASM that the Q3MA-21 is the candidate access-agent, allowing the session transfer and the configuration of the session on the new path by MUSC. Since flows of the requested session are already active on the new path, MUSC only adjusts its state about the number of receivers, allows the configuration of QoS resources on the wireless link and replies to CASM. After the local reply, CASM sends a message to its peer in Q3MA-12. After handover, the fetching of packets from the network cache to the $R 2$ buffer is done as 
explained for $R 1$. In the old access-agent, CASM is triggered to release the cache. On the old path, the state of the session is released by soft-state operations (interaction between MUSC and MIRA). This occurs because the state associated with each flow of $S 1$ is not refreshed by MUSC-P messages before the clean-up time-out expires.

\section{Q3M Evaluation}

Simulations were done using the Network Simulator 2.28 (NS2) to analyze the impact of the Q3M architecture on the expectation of mobile receivers and on the performance of the network. This paper focus on the analysis of the convergence time and signaling overhead of the Q3M components, as well as the impact of the Q3M cache and buffer mechanisms on the user-perceived quality level.

A random topology was generated by Boston University Representative Internet Topology Generator (BRITE) following the same inter-cluster mobility scenario illustrated in Fig. 2. In the topology, each of the three clusters has sixteen routers (four Q3MAs and twelve MIRA interior routers). The intra and inter-cluster links have a bandwidth of $10 \mathrm{Mb} / \mathrm{s}$ and their propagation delay is attributed according to the distance between the edges. Moreover, two receivers are connected to the same access-Q3MA. These receivers get one Variable Bit Rate video with an average rate of $86 \mathrm{~Kb} / \mathrm{s}$. The packet distribution is accomplished by PIM-SSM. Since the usage of mobility prediction is still under investigation, it is assumed that CASM transfers the session context and notifies MUSC in the predicted access-Q3MA before the handover. This allows the pre-configuration of the session in new paths (e.g., prereserve of QoS-aware distribution tree and the cache configuration).

Table I presents the results of the convergence time of the Q3M components before and after the attachment of the moving receivers to the new access-agent. The convergence time before the attachment includes CASM, MUSC and MIRA signaling and their procedures to configure the cache, QoS mapping, adaptation and connectivity, and network service class, respectively. The convergence time after the attachment encompasses CASM operations to fetch the missing packets.

Table 1. CASM, MUSC and MIRA convergence time

\begin{tabular}{c|c|c|c|c|c}
\hline \multirow{2}{*}{$\begin{array}{c}\text { Mobile } \\
\text { Receiver }\end{array}$} & \multicolumn{3}{|c|}{ Before Handover } & After & \multirow{2}{*}{ Total (ms) } \\
\cline { 2 - 5 } R1 & CASM & MUSC & MIRA & CASM & \\
R2 & 24.12 & 13.48 & 10.97 & 1.78 & 50.35 \\
\hline
\end{tabular}

The results demonstrate that CASM requires more time to transfer the sessioncontext and to configure its mechanism than MUSC or MIRA. The MUSC session setup time would be higher if the requested session for $R l$ would be activated in an agent near the source. The MIRA convergence time is small, because only intracluster operations are performed to reserve network resources in the selected class. Since the resources associated with the session requested by $R 2$ are already configured on the path ending in the new access-agent, the MIRA and MUSC convergence time are negligible. Only local operations are done to configure the 
number of receivers, quality level and connectivity functions associated with the session and to reply to CASM. This functionality allows a reduction of $60 \%$ in the overall signaling overhead and $50 \%$ in the time required to setup the same session for a second receiver in the same access-agent. After handover, each receiver needs to wait less than $2 \mathrm{~ms}$ to receive packets, because the session is already installed. This period includes the CASM procedures to fetch the missing packets during handover.

The benefit of the Q3M architecture to recover missing packets when the handover duration varies from 50 to $1000 \mathrm{~ms}$ and considering a buffer size with $100 \mathrm{~KB}$ is presented in Fig. 3. The results show the influence of the Q3M solution on the session quality level achieved by recovering missing packets after handover. For instance, the proposal recovers 47 packets when the handover duration is $500 \mathrm{~ms}$ (including the convergence time of Q3M components) and the cache size is of $50 \mathrm{~KB}$.

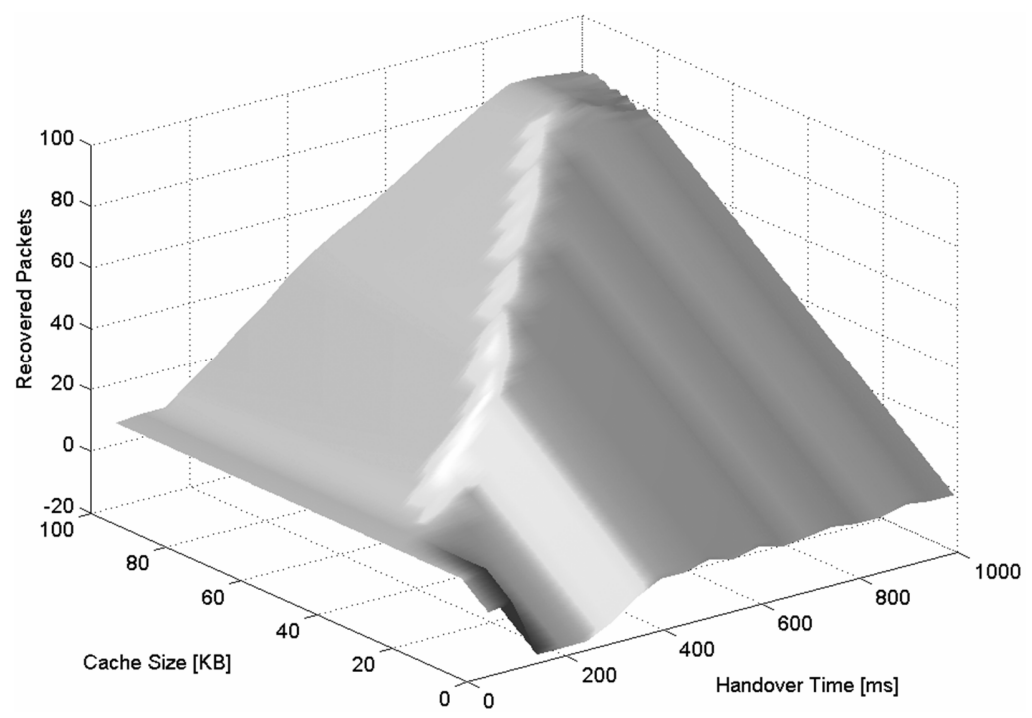

Fig. 3. Recovered packets

\section{Conclusions}

This paper presents the Q3M solution for a unifying IP-based architecture to support seamless multimedia multi-user sessions in $4 \mathrm{G}$ systems. With Q3M architecture, users are unaware of the network technology diversity, and experience seamless mobility. The seamless characteristic of the Q3M solution is achieved by a modular integration of the control of group communication sessions, network resources, and device mobility. By implementing most of the network computation at the edges, a cost reduction is expected. Operators will also be able to provide services with high availability times due to the high robustness of the decentralized architecture.

The evaluation shows that the Q3M architecture has a convergence time and a signaling overhead reduced for a second receiver for the same session in the same access-agent. Moreover, Q3M provides seamless experience to the user by reducing 
the packet losses and latency during handover. Finally, further evaluations to verify the impact of the $\mathrm{Q} 3 \mathrm{M}$ architecture in an experimental environment and the standardization of its protocols as well as the study of mobility prediction schemes to achieve higher seamless handover will be done in future work.

\section{References}

1. Hartung, F., et al.: Advances in Network-Supported Media Delivery in the NextGeneration Mobile Systems, IEEE Communications Magazine 44(8) (August 2006)

2. Greve, F., et al.: FAMOUS: A Network Architecture for Delivering Multimedia Services to FAst MOving USers. In: Wireless Personal Communications, vol. 33(3-4), Kluwer Academic Publishers, Dordrecht (2005)

3. Rojan, G., et al.: Policy Based QoS Architecture in MUSE. In: Proc. of Annual IEEE Conference on Computer Communications, Barcelona, Madrid (April 2006)

4. Miloucheva, I., et al.: QoS Based Multicast Architecture for Heterogeneous Mobile IPv6 Environment. In: Proc. International Conference on Telecommunication, Madeira, Portugal (May 2006)

5. Dugeon, O., et al.: End-to-End Quality of Service over Heterogeneous Networks (EuQoS). In: Proc. of Fourth IFIP International Conference on Network Control and Engineering for QoS, Security and Mobility, Lannion, France (November 2005)

6. Jamalipour, A., Mirchandani, V., Kibria, M.: QoS-aware Mobility Support Architecture for Next generation Network, Wireless Communications \& Mobile Computing 5(8) (December 2005)

7. Cerqueira, E., et al.: Multi-user Session Control in the Next Generation Wireless System. In: Proc. of ACM Workshop on Mobile Management and Wireless Access, Malaga, Spain (October 2006)

8. Neto, A., et al.: Resource Reservation Protocol Supporting QoS-aware Multicast Trees for Next Generation Networks. In: Proc. of IEEE Symposium Computer and Communications, Aveiro Portugal (July 2007)

9. Veloso, L., et al.: Seamless Mobility of Users for Media Distribution Services. In: Proc. of IEEE Performance Computing and Communications Conference, New Orleans, USA (April 2007)

10. Cicconetti, C., et al.: Quality of Service Support in IEEE 802.16 Networks, IEEE Network 20(5) (March-April 2006)

11. Sricharan, M., et al.: An Activity Based Mobility Prediction Strategy for Next Generation Wireless Networks. In: Proc. of IFIP Conference on Wireless and Optical Communications Networks, Bangalore, India (April 2006) 